\title{
On the Stability of a Functional Equation Associated with the Fibonacci Numbers
}

\author{
Cristinel Mortici, ${ }^{1,2}$ Michael Th. Rassias, ${ }^{3}$ and Soon-Mo Jung ${ }^{4}$ \\ ${ }^{1}$ Valahia University of Târgovişte, Bulevardul Unirii 18, 130082 Târgoviște, Romania \\ ${ }^{2}$ Academy of Romanian Scientists, Splaiul Independenţei 54, 050094 Bucharest, Romania \\ ${ }^{3}$ Department of Mathematics, ETH Zürich, Raemistrasse 101, 8092 Zürich, Switzerland \\ ${ }^{4}$ Mathematics Section, College of Science and Technology, Hongik University, Sejong 339-701, Republic of Korea
}

Correspondence should be addressed to Soon-Mo Jung; smjung@hongik.ac.kr

Received 5 May 2014; Accepted 8 July 2014; Published 20 July 2014

Academic Editor: Chengjian Zhang

Copyright (c) 2014 Cristinel Mortici et al. This is an open access article distributed under the Creative Commons Attribution License, which permits unrestricted use, distribution, and reproduction in any medium, provided the original work is properly cited.

We prove the Hyers-Ulam stability of the generalized Fibonacci functional equation $F(x)-g(x) F(h(x))=0$, where $g$ and $h$ are given functions.

\section{Introduction}

In 1940, Ulam [1] gave a wide ranging talk before the mathematics club of the University of Wisconsin in which he discussed a number of important unsolved problems. Among them was the question concerning the stability of group homomorphisms.

Let $G_{1}$ be a group and let $G_{2}$ be a metric group with the metric $d(\cdot, \cdot)$. Given $\varepsilon>0$, does there exist a $\delta>0$ such that if a function $h: G_{1} \rightarrow G_{2}$ satisfies the inequality $d(h(x y), h(x) h(y))<\delta$, for all $x, y \in G_{1}$, then there exists a homomorphism $H: G_{1} \rightarrow G_{2}$ with $d(h(x), H(x))<\varepsilon$, for all $x \in G_{1}$ ?

The case of approximately additive functions was solved by Hyers [2] under the assumption that $G_{1}$ and $G_{2}$ are Banach spaces. Indeed, he proved the following theorem.

Theorem 1. Let $f: G_{1} \rightarrow G_{2}$ be a function between Banach spaces such that

$$
\|f(x+y)-f(x)-f(y)\| \leq \varepsilon,
$$

for some $\varepsilon>0$ and for all $x, y \in G_{1}$. Then, the limit

$$
A(x)=\lim _{n \rightarrow \infty} 2^{-n} f\left(2^{n} x\right)
$$

exists for each $x \in G_{1}$, and $A: G_{1} \rightarrow G_{2}$ is the unique additive function such that

$$
\|f(x)-A(x)\| \leq \varepsilon,
$$

for any $x \in G_{1}$. Moreover, if $f(t x)$ is continuous in $t$, for each fixed $x \in G_{1}$, then the function $A$ is linear.

Hyers proved that each solution of the inequality $\| f(x+$ $y)-f(x)-f(y) \| \leq \varepsilon$ can be approximated by an exact solution; say an additive function. In this case, the Cauchy additive functional equation, $f(x+y)=f(x)+f(y)$, is said to have the Hyers-Ulam stability.

Since then, the stability problems of a large variety of functional equations have been extensively investigated by several mathematicians (cf. [3-14]).

In this paper, we investigate the Hyers-Ulam stability of the functional equation

$$
F(x)-g(x) F(h(x))=0,
$$

where $g$ and $h$ are given functions.

In Section 2, we prove that the functional equation (4) has a large class of nontrivial solutions. Section 3 is devoted to the investigation of the Hyers-Ulam stability problems for (4). In the last section, we prove the Hyers-Ulam stability of (4) when $g$ is a constant function, which is a generalization 
of the papers $[4,7,14]$. More precisely, Jung [7] proved the Hyers-Ulam stability of the generalized Fibonacci functional equation

$$
f(x)=p f(x-1)-q f(x-2)
$$

in the class of functions $f: \mathbb{R} \rightarrow X$, where $X$ is a real (or complex) Banach space.

Theorem 2 (see [7, Theorem 3.1]). Assume that the quadratic equation $x^{2}-p x+q=0$ has real solutions $a$ and $b$ with $0<$ $|b|<1<|a|$. If a function $f: \mathbb{R} \rightarrow X$ satisfies the inequality

$$
\|f(x)-p f(x-1)+q f(x-2)\| \leq \varepsilon
$$

for all $x \in \mathbb{R}$ and for some $\varepsilon>0$, then there exists a unique solution $F: \mathbb{R} \rightarrow X$ of (5) such that

$$
\|f(x)-F(x)\| \leq \frac{|a|-|b|}{|a-b|} \cdot \frac{\varepsilon}{(|a|-1)(1-|b|)}
$$

for all $x \in \mathbb{R}$.

A similar case for $0<|b|<1<|a|$ with $|b| \neq 1 / 2$ was investigated by Brzdęk et al. [4] and Trif [14] who obtained the estimate

$$
\|f(x)-F(x)\| \leq \frac{4 \varepsilon}{|2| b|-1| \cdot(2|a|-1)} .
$$

If either $0<|b|<1 / 2$ and $|a|>3 / 2-|b|$ or $1 / 2<|b|<3 / 4$ and $|a|>(5-6|b|) /(6-8|b|)$, then the inequality (7) is sharper than that of (8).

In Section 4 of this paper, we improve the results of papers $[4,7,14]$ in the sense that we estimate $\|f(x)-F(x)\|$ even when both $|a|$ and $|b|$ are larger or smaller than 1. Moreover, we deal with a functional equation (4) that is regarded as a more generalized form of the Fibonacci functional equation (5).

In this paper, $\mathbb{R}, \mathbb{Z}$, and $\mathbb{N}$ stand for the sets of real numbers, integers, and positive integers, respectively.

\section{Solutions of $(4)$}

Evidently, (4) admits the trivial solution $F=0$. In order to avoid the trivial case, we search in this section for a class of nontrivial solutions of (4).

Let $D$ be a subset of $\mathbb{R}$. A function $h: D \rightarrow D$ is said to be of disjoint iterated images, shortly (DII)-function, if

(i) there exists a partition

$$
D=\coprod_{n \geq 1} D_{n}
$$

(ii) $h$ maps bijectively $D_{n}$ onto $D_{n+1}$ for each integer $n \geq 1$.

As an example for a (DII)-function, we introduce a function $h:(0,1] \rightarrow(0,1]$ defined by

$$
h(x)=\frac{1}{n+2}\left(n x+\frac{1}{n+1}\right), \quad x \in D_{n}:=\left(\frac{1}{n+1}, \frac{1}{n}\right],
$$

for all $n \in \mathbb{N}$. For every $n \in \mathbb{N}$, this function is linear on $D_{n}$ and it transforms each $D_{n}$ onto $D_{n+1}$.

We are now in a position to prove that the set of all solutions of (4) is not empty but it is an infinite set.

Theorem 3. Leth $: D \rightarrow D$ be a (DII)-function and $g: D \rightarrow$ $\mathbb{R} \backslash\{0\}$. There is a one-to-one correspondence between the set of all solutions $F: D \rightarrow \mathbb{R}$ of the functional equation (4) and the set of all functions $\varphi: D_{1} \rightarrow \mathbb{R}$.

Proof. Given a $\varphi: D_{1} \rightarrow \mathbb{R}$, we define a function $F$ on $D_{1}$ as

$$
F(x)=\varphi(x),
$$

for all $x \in D_{1}$. Assume that $F$ is defined on $D_{n-1}$ for some $n \geq 2$. If $x \in D_{n}$, then $h^{-1}(x) \in D_{n-1}$ and we put

$$
F(x)=\frac{1}{g\left(h^{-1}(x)\right)} F\left(h^{-1}(x)\right),
$$

for all $x \in D_{n}$. By this inductive procedure, $F$ is completely defined.

We now show that $F$ is a solution of (4). Let $z$ be any point of $D$ and let $n \geq 2$ be an integer such that $h(z) \in D_{n}$. Put $x=h(z)$ in (12) to get

$$
F(h(z))=\frac{1}{g(z)} F(z),
$$

which is (4).

Conversely, we associate to every solution $F$ of (4) the function $\varphi=\left.F\right|_{D_{1}}$.

We notice that a (DII)-function $h$ is injective as we see the following: if $x, y \in D_{n}$ for some $n \in \mathbb{N}$ with $x \neq y$ but $h(x)=$ $h(y)$, then $h(x)=h(y) \in D_{n+1}$ and, hence, $x=y$ because $h$ maps bijectively $D_{n}$ onto $D_{n+1}$, a contradiction. If $x \in D_{m}$ and $y \in D_{n}$ for some $m, n \in \mathbb{N}$ with $m \neq n$, it is then obvious that $h(x) \neq h(y)$ because $h(x) \in D_{m+1}, h(y) \in D_{n+1}$, and $D_{m+1} \cap D_{n+1}=\emptyset$. But $h$ is not surjective, since $I m h=D \backslash D_{1}$.

We now study the set of solutions of (4) under the assumption that $h: D \rightarrow D$ is a bijection. For any pair of points $x, y \in D$, we use the notation $x=y$ if there exists a $k \in \mathbb{Z}$ with $y=h^{k}(x)$. Since " $\asymp$ " is an equivalence relation in $D$, let

$$
D=\coprod_{i \in I} \Delta_{i}
$$

be the corresponding partition in “ $\asymp$-equivalence classes" $\Delta_{i}=\widehat{x}_{i}(i \in I)$; that is,

$$
\Delta_{i}=\left\{h^{k}\left(x_{i}\right) \mid k \in \mathbb{Z}\right\}
$$

Theorem 4. Given a subset $D$ of $\mathbb{R}$, let $h: D \rightarrow D$ be a bijective function and $g: D \rightarrow \mathbb{R} \backslash\{0\}$. Assume that

$$
D=\coprod_{i \in I} \Delta_{i}
$$


is a partition of $D$ corresponding to the equivalence relation $\asymp$ with the property (15). Then, there exists a one-to-one correspondence between the set of all solutions $F: D \rightarrow \mathbb{R}$ of the functional equation (4) and the set of all real sequences $\left\{y_{i}\right\}_{i \in I}$.

Proof. For any real sequence $\left\{y_{i}\right\}_{i \in I}$, we define $F\left(x_{i}\right)=y_{i}$ for all $i \in I$, where $I$ is the index set for the partition corresponding to the equivalence relation $\approx$ with the property (15). We further define the function $F: D \rightarrow \mathbb{R}$ by

$$
\begin{gathered}
F\left(h\left(x_{i}\right)\right)=\frac{1}{g\left(x_{i}\right)} F\left(x_{i}\right)=\frac{1}{g\left(x_{i}\right)} y_{i}, \\
F\left(h^{-1}\left(x_{i}\right)\right)=g\left(h^{-1}\left(x_{i}\right)\right) F\left(x_{i}\right)=g\left(h^{-1}\left(x_{i}\right)\right) y_{i} .
\end{gathered}
$$

In general, if $F$ is defined at $h^{k}\left(x_{i}\right)$ and $h^{-k}\left(x_{i}\right)$, then $F$ is defined at $h^{k+1}\left(x_{i}\right)$ and $h^{-k-1}\left(x_{i}\right)$ by

$$
\begin{gathered}
F\left(h^{k+1}\left(x_{i}\right)\right)=\frac{1}{g\left(h^{k}\left(x_{i}\right)\right)} F\left(h^{k}\left(x_{i}\right)\right), \\
F\left(h^{-k-1}\left(x_{i}\right)\right)=g\left(h^{-k-1}\left(x_{i}\right)\right) F\left(h^{-k}\left(x_{i}\right)\right) .
\end{gathered}
$$

For each $i \in I$, we can use such an inductive procedure to define the function $F$ on $\Delta_{i}$ and we see that $\left.F\right|_{\Delta_{i}}$ is uniquely determined by the value of $y_{i}$.

Conversely, every solution $F: D \rightarrow \mathbb{R}$ of (4) can be associated to the real sequence $\left\{F\left(x_{i}\right)\right\}_{i \in I}$.

Corollary 5. Given a subset $D$ of $\mathbb{R}$, let $h: D \rightarrow D$ be a bijective function and $\alpha \in \mathbb{R} \backslash\{0\}$. Assume that

$$
D=\coprod_{i \in I} \Delta_{i}
$$

is a partition of $D$ corresponding to the equivalence relation $\asymp$ with the property (15). Then there exists a one-to-one correspondence between the set of all solutions $F: D \rightarrow \mathbb{R}$ of the functional equation

$$
F(x)-\alpha F(h(x))=0
$$

and the set of all real numbers $\left\{y_{i}\right\}_{i \in I}$.

\section{Hyers-Ulam Stability of (4)}

The above conditions imposed on the function $h$ were necessary for showing that the functional equations (4) and (20) have large classes of nontrivial solutions. The stability results presented in the sequel are valid also under weaker conditions as we shall see in the following theorems.

Theorem 6. Given real numbers $a$ and $b$ with $a<b$, let $h$ : $(a, b) \rightarrow(a, b)$ and $g:(a, b) \rightarrow I$ be given functions, where $I \subset(0,1)$ is an interval of length $l$. Assume that a bounded function $f:(a, b) \rightarrow \mathbb{R}$ satisfies the inequality

$$
|f(x)-g(x) f(h(x))| \leq \varepsilon
$$

for all $x \in(a, b)$ and for some $\varepsilon>0$. Then, for every $\alpha \in I$, there exists a solution $F:(a, b) \rightarrow \mathbb{R}$ of $(20)$ such that

$$
|f(x)-F(x)| \leq \frac{\varepsilon+l|f|_{\infty}}{1-\alpha}
$$

for any $x \in(a, b)$, where $|f|_{\infty}=\sup _{x \in(a, b)}|f(x)|$.

Proof. First, we prove that

$$
|f(x)-\alpha f(h(x))| \leq \lambda,
$$

for all $x \in(a, b)$, where we set $\lambda=\varepsilon+l|f|_{\infty}$. Indeed, it follows from (21) that

$$
\begin{aligned}
& |f(x)-\alpha f(h(x))| \\
& \quad \leq|f(x)-g(x) f(h(x))|+|g(x) f(h(x))-\alpha f(h(x))| \\
& \quad \leq \varepsilon+|g(x)-\alpha| \cdot|f(h(x))| \\
& \quad \leq \varepsilon+l|f|_{\infty} \\
& \quad=\lambda,
\end{aligned}
$$

for every $x \in(a, b)$.

By replacing $x$ with $h^{k}(x)$ and then multiplying with $\alpha^{k}$ both sides of (23), we get

$$
\left|\alpha^{k} f\left(h^{k}(x)\right)-\alpha^{k+1} f\left(h^{k+1}(x)\right)\right| \leq \lambda \alpha^{k},
$$

for all $x \in(a, b)$ and $k \in \mathbb{N}$. Since

$$
\begin{aligned}
& \left|f(x)-\alpha^{n} f\left(h^{n}(x)\right)\right| \\
& \quad \leq \sum_{k=0}^{n-1}\left|\alpha^{k} f\left(h^{k}(x)\right)-\alpha^{k+1} f\left(h^{k+1}(x)\right)\right| \\
& \quad \leq \sum_{k=0}^{n-1} \lambda \alpha^{k}=\lambda \frac{1-\alpha^{n}}{1-\alpha},
\end{aligned}
$$

we have

$$
\left|f(x)-\alpha^{n} f\left(h^{n}(x)\right)\right| \leq \lambda \frac{1-\alpha^{n}}{1-\alpha},
$$

for any $x \in(a, b)$ and $n \in \mathbb{N}$.

The inequality (25) shows that the sequence $\left\{\alpha^{n} f\left(h^{n}(x)\right)\right\}_{n \in \mathbb{N}}$ is a Cauchy sequence for every $x \in(a, b)$. Thus, we can define a function $F:(a, b) \rightarrow \mathbb{R}$ by

$$
F(x)=\lim _{n \rightarrow \infty} \alpha^{n} f\left(h^{n}(x)\right),
$$

for all $x \in(a, b)$. Hence, it follows from (23) that

$$
\begin{aligned}
\mid F & (x)-\alpha F(h(x)) \mid \\
& =\left|\lim _{n \rightarrow \infty} \alpha^{n} f\left(h^{n}(x)\right)-\lim _{n \rightarrow \infty} \alpha^{n+1} f\left(h^{n+1}(x)\right)\right| \\
& =\lim _{n \rightarrow \infty}\left|\alpha^{n}\left(f\left(h^{n}(x)\right)-\alpha f\left(h^{n+1}(x)\right)\right)\right| \\
& \leq \lim _{n \rightarrow \infty} \alpha^{n} \lambda=0,
\end{aligned}
$$

for each $x \in(a, b)$, which implies that $F$ is a solution of (20).

Finally, inequality (22) is an immediate consequence of (27) if we take the limit as $n \rightarrow \infty$. 


\section{When $g$ Is Constant}

In the case of $g(x)=s \notin\{-1,+1\}$ for all $x \in \mathbb{R}$, we investigate the Hyers-Ulam stability of the functional equation

$$
F(x)-s F(h(x))=0,
$$

where $h: \mathbb{R} \rightarrow \mathbb{R}$ and $F: \mathbb{R} \rightarrow X$ are functions and $X$ is a real Banach space.

Theorem 7. Let $X$ be a real Banach space and let $s$ be a real number with $|s|<1$. If a function $f: \mathbb{R} \rightarrow X$ satisfies the inequality

$$
\|f(x)-s f(h(x))\| \leq \varepsilon,
$$

for all $x \in \mathbb{R}$ and for some $\varepsilon>0$, then there exists a solution $F: \mathbb{R} \rightarrow X$ of (30) such that

$$
\|f(x)-F(x)\| \leq \frac{\varepsilon}{1-|s|},
$$

for all $x \in \mathbb{R}$.

Proof. By replacing $x$ with $h^{k}(x)$ and multiplying with $s^{k}$ both sides of (31), we get

$$
\left\|s^{k} f\left(h^{k}(x)\right)-s^{k+1} f\left(h^{k+1}(x)\right)\right\| \leq \varepsilon|s|^{k},
$$

for all $x \in \mathbb{R}$ and $k \in\{0,1,2, \ldots\}$. By (33), we have

$$
\begin{aligned}
& \left\|f(x)-s^{n} f\left(h^{n}(x)\right)\right\| \\
& \leq \sum_{k=0}^{n-1}\left\|s^{k} f\left(h^{k}(x)\right)-s^{k+1} f\left(h^{k+1}(x)\right)\right\| \\
& \quad \leq \sum_{k=0}^{n-1} \varepsilon|s|^{k}=\varepsilon \frac{1-|s|^{n}}{1-|s|}
\end{aligned}
$$

for all $x \in \mathbb{R}$ and $n \in \mathbb{N}$. Hence, we get

$$
\left\|f(x)-s^{n} f\left(h^{n}(x)\right)\right\| \leq \varepsilon \frac{1-|s|^{n}}{1-|s|},
$$

for any $x \in \mathbb{R}$ and $n \in \mathbb{N}$.

The inequality (33) shows that the sequence $\left\{s^{n} f\left(h^{n}(x)\right)\right\}_{n \in \mathbb{N}}$ is a Cauchy sequence for any fixed $x \in \mathbb{R}$. Thus, since $X$ is a complete space, we can define a function $F: \mathbb{R} \rightarrow X$ by

$$
F(x)=\lim _{n \rightarrow \infty} s^{n} f\left(h^{n}(x)\right)
$$

for all $x \in \mathbb{R}$.

It follows from (31) that

$$
\begin{aligned}
\| F & (x)-s F(h(x)) \| \\
& =\left\|\lim _{n \rightarrow \infty} s^{n} f\left(h^{n}(x)\right)-\lim _{n \rightarrow \infty} s^{n+1} f\left(h^{n+1}(x)\right)\right\| \\
& =\lim _{n \rightarrow \infty} s^{n}\left\|f\left(h^{n}(x)\right)-s f\left(h^{n+1}(x)\right)\right\| \\
& \leq \lim _{n \rightarrow \infty} s^{n} \varepsilon=0,
\end{aligned}
$$

which implies that $F$ is a solution of (30).

Finally, the inequality (32) immediately follows from (35) provided that we take the limit as $n \rightarrow \infty$.
Assume now that $h: \mathbb{R} \rightarrow \mathbb{R}$ is bijective. A similar theorem can be proved when $|s|>1$.

Theorem 8. Let $X$ be a real Banach space, let $h: \mathbb{R} \rightarrow \mathbb{R}$ be a bijective function, and let $s \in \mathbb{R}$ be given with $|s|>1$. If a function $f: \mathbb{R} \rightarrow X$ satisfies the inequality

$$
\|f(x)-s f(h(x))\| \leq \varepsilon,
$$

for all $x \in \mathbb{R}$ and for some $\varepsilon>0$, then there exists a solution $F: \mathbb{R} \rightarrow X$ of (30) such that

$$
\|f(x)-F(x)\| \leq \frac{\varepsilon}{|s|-1}
$$

for all $x \in \mathbb{R}$.

Proof. By replacing $x$ with $h^{-1}(x)$ and dividing by $s$ both sides of (38), we get

$$
\left\|f(x)-\frac{1}{s} f\left(h^{-1}(x)\right)\right\| \leq \frac{\varepsilon}{|s|},
$$

for any $x \in \mathbb{R}$. Since the constant $1 /|s|$ is less than 1 , our assertion follows from Theorem 7 . In particular, we have

$$
F(x)=\lim _{n \rightarrow \infty} s^{-n} f\left(h^{-n}(x)\right),
$$

for each $x \in \mathbb{R}$.

Corollary 9. Let $X$ be a real Banach space, let $h: \mathbb{R} \rightarrow \mathbb{R}$ be a bijective function, and let $s \in \mathbb{R}$ be given with $|s| \neq 1$. If a function $f: \mathbb{R} \rightarrow X$ satisfies the inequality

$$
\|f(x)-s f(h(x))\| \leq \varepsilon,
$$

for any $x \in \mathbb{R}$ and for some $\varepsilon>0$, then there exists a solution $F: \mathbb{R} \rightarrow X$ of (30) such that

$$
\|f(x)-F(x)\| \leq \frac{\varepsilon}{|| s|-1|},
$$

for all $x \in \mathbb{R}$.

By combining the results of Theorems 7 and 8 , we can present a stability result of the following functional equation

$$
F(x)-p F(h(x))+q F(h(h(x)))=0,
$$

where $h: \mathbb{R} \rightarrow \mathbb{R}$ is bijective and the range space of the function $F: \mathbb{R} \rightarrow X$ is a real Banach space.

Theorem 10. Let $p$ and $q$ be given real numbers such that the quadratic equation $x^{2}-p x+q=0$ has distinct real solutions a and $b$ with $|a| \neq 1$ and $|b| \neq 1$. Assume that a bijective function $h: \mathbb{R} \rightarrow \mathbb{R}$ is given and $X$ is a real Banach space. If a function $f: \mathbb{R} \rightarrow X$ satisfies

$$
\|f(x)-p f(h(x))+q f(h(h(x)))\| \leq \varepsilon,
$$

for all $x \in \mathbb{R}$ and for some $\varepsilon>0$, then there exists a solution $F: \mathbb{R} \rightarrow X$ of (44) such that

$$
\|f(x)-F(x)\| \leq \frac{1}{|a-b|}\left(\frac{1}{\left.|| a\right|^{-1}-1 \mid}+\frac{1}{\left.|| b\right|^{-1}-1 \mid}\right) \varepsilon,
$$

for all $x \in \mathbb{R}$. 
As we mentioned in the Introduction, our result extends Jung's result in [7], since

$$
\begin{array}{r}
\frac{1}{|a-b|}\left(\frac{1}{\left.|| a\right|^{-1}-1 \mid}+\frac{1}{\left.|| b\right|^{-1}-1 \mid}\right) \varepsilon \\
\quad=\frac{|a|-|b|}{|a-b|} \cdot \frac{\varepsilon}{(|a|-1)(1-|b|)}
\end{array}
$$

when $0<|b|<1<|a|$. Moreover, Jung's result is a particular case of Theorem 10 when we set $h(x)=x-1$ in (45).

\section{Proof of Theorem 10. If we set}

$$
u(x)=f(x)-a f(h(x))
$$

then the inequality (45) yields

$$
\|u(x)-b u(h(x))\| \leq \varepsilon,
$$

for all $x \in \mathbb{R}$. According to Corollary 9 , there exists a solution $U: \mathbb{R} \rightarrow X$ of

$$
U(x)-b U(h(x))=0
$$

with

$$
\|u(x)-U(x)\| \leq \frac{\varepsilon}{|| b|-1|},
$$

for any $x \in \mathbb{R}$.

If we set

$$
v(x)=f(x)-b f(h(x))
$$

then the inequality (45) yields

$$
\|v(x)-a v(h(x))\| \leq \varepsilon,
$$

for any $x \in \mathbb{R}$. In view of Corollary 9 again, there exists a solution $V: \mathbb{R} \rightarrow X$ of

$$
V(x)-a V(h(x))=0
$$

with

$$
\|v(x)-V(x)\| \leq \frac{\varepsilon}{|| a|-1|},
$$

for all $x \in \mathbb{R}$.

We now define a function $F: \mathbb{R} \rightarrow X$ by

$$
F_{\lambda}(x)=(1-\lambda) U(x)+\lambda V(x),
$$

for any $x \in \mathbb{R}$, where $\lambda$ is a real number. Then, it follows from (50) and (54) that

$$
\begin{aligned}
& F_{\lambda}(x)-p F_{\lambda}(h(x))+q F_{\lambda}(h(h(x))) \\
&= F_{\lambda}(x)-b F_{\lambda}(h(x))-a\left(F_{\lambda}(h(x))-b F_{\lambda}(h(h(x)))\right) \\
&=(1-\lambda)(U(x)-b U(h(x)) \\
&-a(U(h(x))-b U(h(h(x))))) \\
&+\lambda(V(x)-b V(h(x))-a(V(h(x))-b V(h(h(x))))) \\
&= \lambda(V(x)-b V(h(x))-a(V(h(x))-b V(h(h(x))))) \\
&= \lambda\left(\left(1-\frac{b}{a}\right) V(x)-a\left(1-\frac{b}{a}\right) V(h(x))\right) \\
&= \lambda\left(1-\frac{b}{a}\right)(V(x)-a V(h(x)))=0,
\end{aligned}
$$

for all $x \in \mathbb{R}$, which implies that $F_{\lambda}$ is a solution of (44) for every fixed real number $\lambda$.

We now set

$$
\lambda=\frac{-a}{b-a}
$$

and assert that the function

$$
F(x)=\frac{b}{b-a} U(x)-\frac{a}{b-a} V(x)
$$

satisfies the requirements of this theorem. Indeed, it follows from (51) and (55) that

$$
\begin{aligned}
&\left\|f(x)-\left(\frac{b}{b-a} U(x)-\frac{a}{b-a} V(x)\right)\right\| \\
&=\frac{1}{|b-a|}\|(b-a) f(x)-(b U(x)-a V(x))\| \\
& \leq \frac{|b|}{|b-a|}\|U(x)-(f(x)-a f(h(x)))\| \\
&+\frac{|a|}{|b-a|}\|V(x)-(f(x)-b f(h(x)))\| \\
& \leq \frac{|b|}{|b-a|}\|U(x)-u(x)\|+\frac{|a|}{|b-a|}\|V(x)-v(x)\| \\
& \leq \frac{|b|}{|b-a|} \cdot \frac{\varepsilon}{|| b|-1|}+\frac{|a|}{|b-a|} \cdot \frac{\varepsilon}{|| a|-1|} \\
&= \frac{1}{|a-b|}\left(\frac{1}{\left.|| a\right|^{-1}-1 \mid}+\frac{1}{\left.|| b\right|^{-1}-1 \mid}\right) \varepsilon,
\end{aligned}
$$

for any $x \in \mathbb{R}$.

\section{Conflict of Interests}

The authors declare that there is no conflict of interests regarding the publication of this paper. 


\section{Acknowledgments}

Soon-Mo Jung was supported by the Basic Science Research Program through the National Research Foundation of Korea (NRF) funded by the Ministry of Education (no. 2013R1A1A2005557). The work of Cristinel Mortici was supported by a Grant of the Romanian National Authority for Scientific Research, CNCS-UEFISCDI Project no. PNII-ID-PCE-2011-3-0087. Cristinel Mortici's final remarks for improving this paper were made during his visit at CERN laboratories in Geneva, Switzerland.

\section{References}

[1] S. M. Ulam, A Collection of Mathematical Problems, Interscience, New York, NY, USA, 1960.

[2] D. H. Hyers, "On the stability of the linear functional equation," Proceedings of the National Academy of Sciences of the United States of America, vol. 27, pp. 222-224, 1941.

[3] J. Brzdęk and S.-M. Jung, "A note on stability of a linear functional equation of second order connected with the Fibonacci numbers and Lucas sequences," Journal of Inequalities and Applications, vol. 2010, Article ID 793947, 10 pages, 2010.

[4] J. Brzdęk, D. Popa, and B. Xu, "Note on nonstability of the linear recurrence," Abhandlungen aus dem Mathematischen Seminar der Universität Hamburg, vol. 76, pp. 183-189, 2006.

[5] J. Brzdęk, D. Popa, and B. Xu, "Hyers-Ulam stability for linear equations of higher orders," Acta Mathematica Hungarica, vol. 120, no. 1-2, pp. 1-8, 2008.

[6] S. Czerwik, Functional Equations and Inequalities in Several Variables, World Scientific, London, UK, 2002.

[7] S.-M. Jung, "Functional equation $f(x)=p f(x-1)-q f(x-$ 2) and its Hyers-Ulam stability," Journal of Inequalities and Applications, vol. 2009, Article ID 181678, 10 pages, 2009.

[8] S.-M. Jung, "Hyers-Ulam stability of Fibonacci functional equation," Bulletin: Iranian Mathematical Society, vol. 35, no. 2, pp. 217-227, 2009.

[9] S.-M. Jung, D. Popa, and M. T. Rassias, "On the stability of the linear functional equation in a single variable on complete metric groups," Journal of Global Optimization, vol. 59, no. 1, pp. 165-171, 2014.

[10] S.-M. Jung and M. T. Rassias, "A linear functional equation of third order associated with the Fibonacci numbers," Abstract and Applied Analysis, vol. 2014, Article ID 137468, 7 pages, 2014.

[11] M. Kuczma, Functional Equations in a Single Variable, Polish Scientific, Warszawa, Poland, 1968.

[12] Y.-H. Lee, S.-M. Jung, and M. T. Rassias, "On an $n$-dimensional mixed type additive and quadratic functional equation," Applied Mathematics and Computation, vol. 228, pp. 13-16, 2014.

[13] D. Popa, "Hyers-Ulam stability of the linear recurrence with constant coefficients," Advances in Difference Equations, no. 2, pp. 101-107, 2005.

[14] T. Trif, "Hyers-Ulam-Rassias stability of a linear functional equation with constant coefficients," Nonlinear Functional Analysis and Applications, vol. 11, no. 5, pp. 881-889, 2006. 


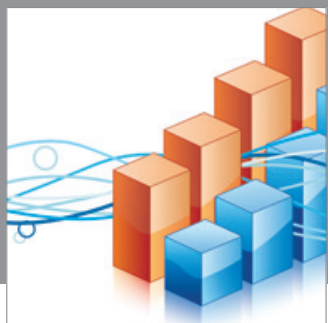

Advances in

Operations Research

mansans

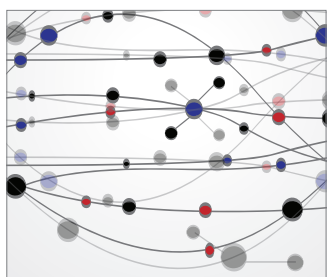

The Scientific World Journal
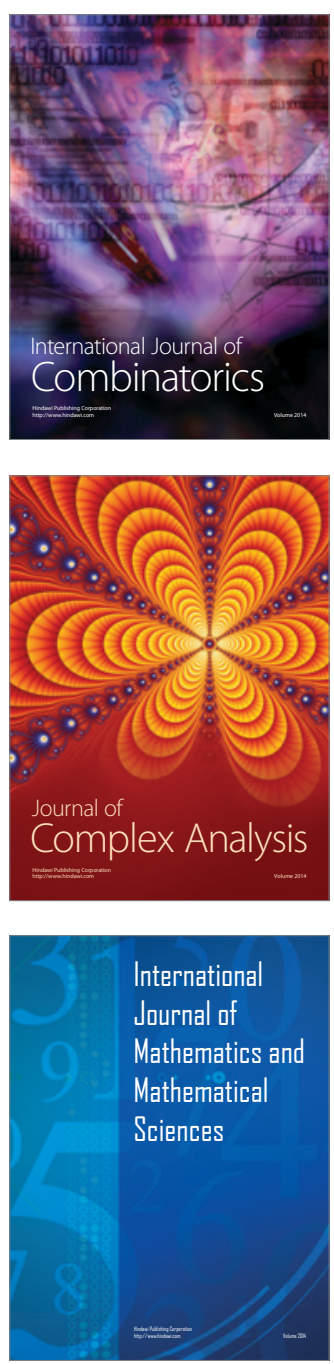
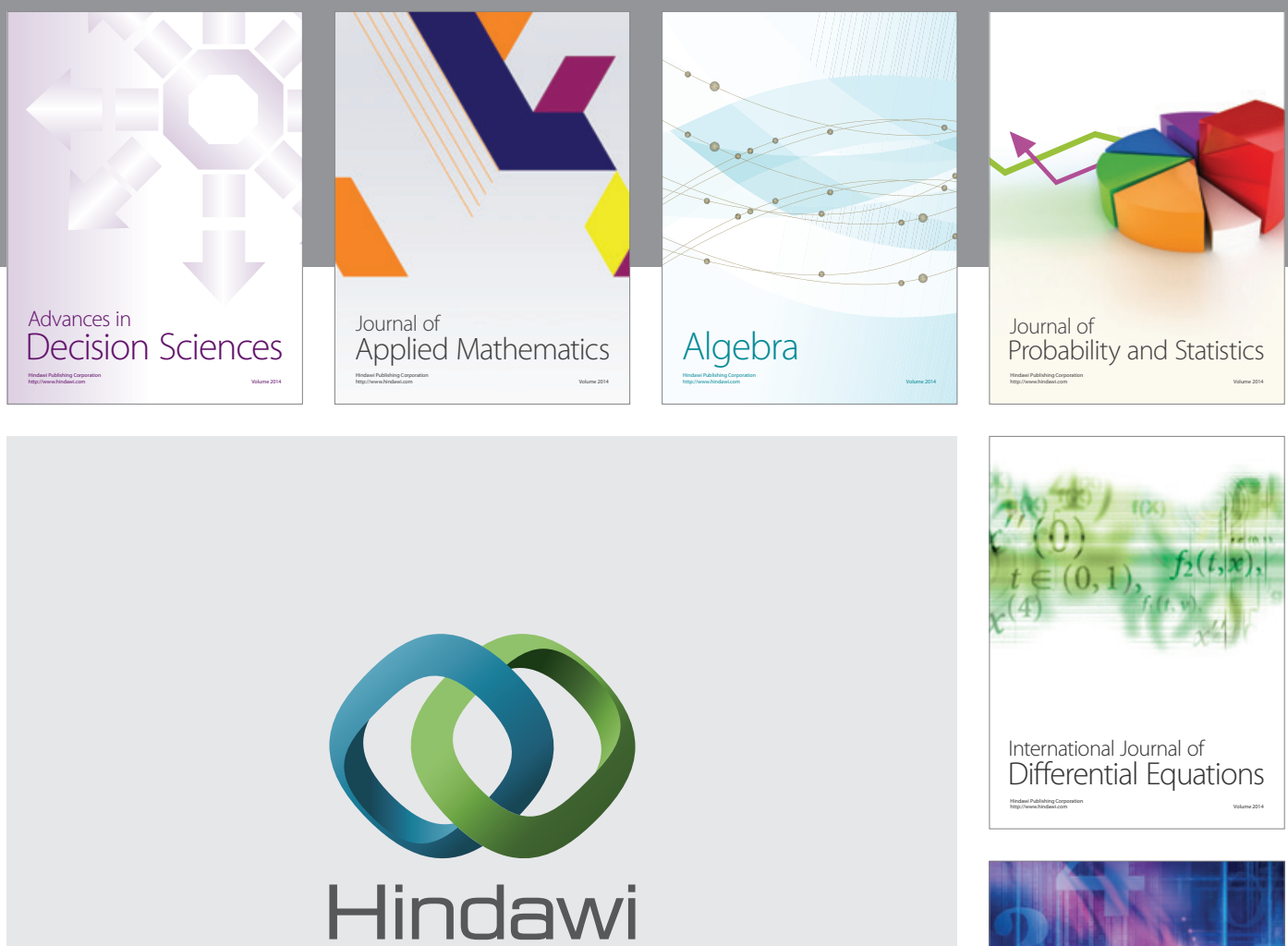

Submit your manuscripts at http://www.hindawi.com
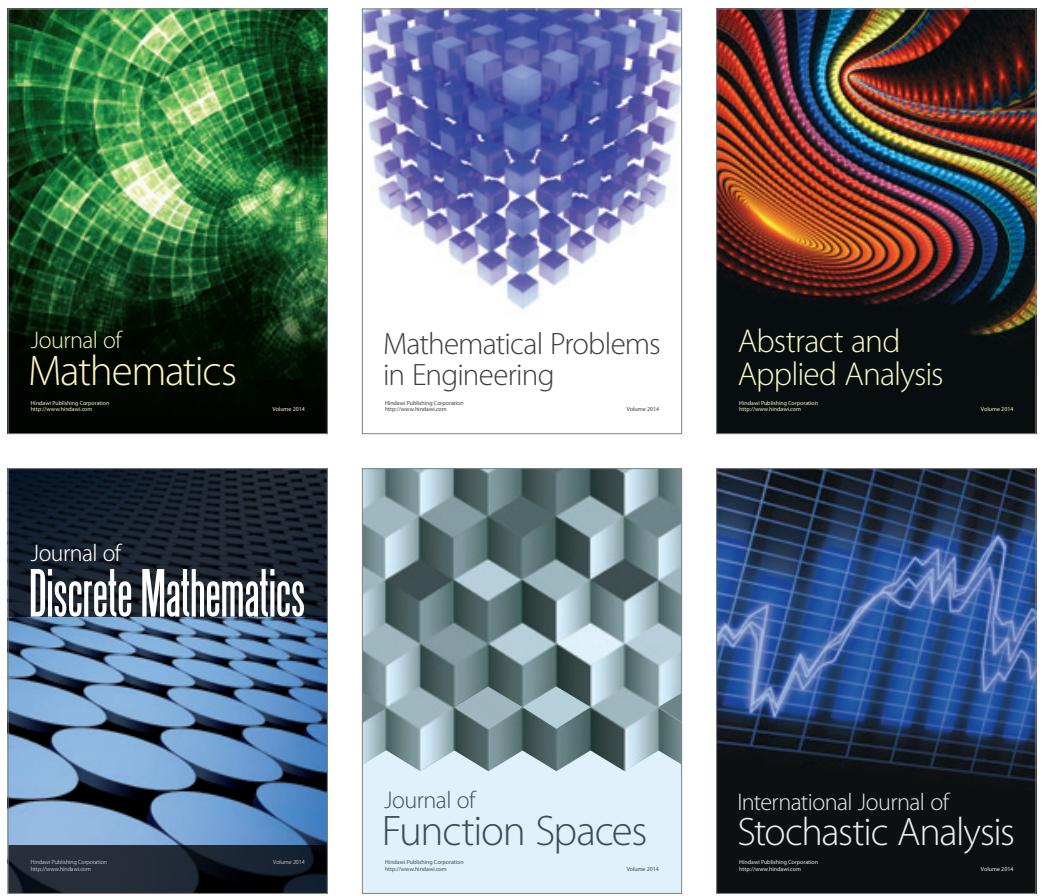

Journal of

Function Spaces

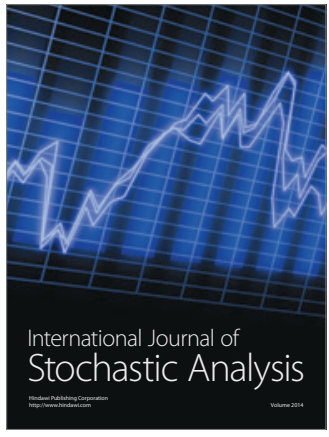

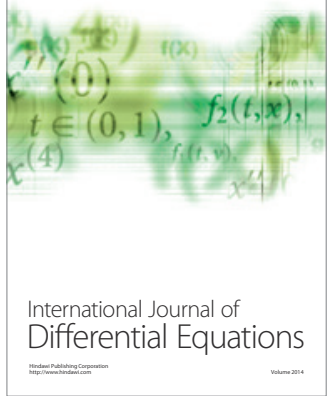
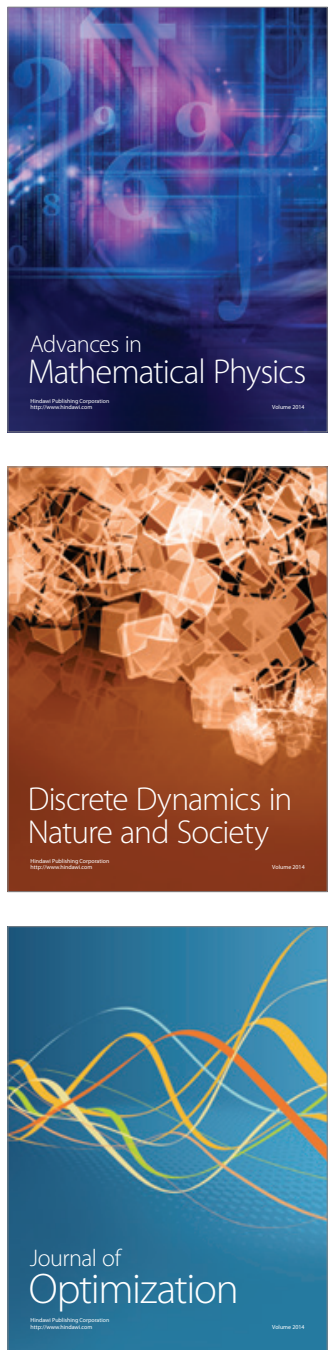